\title{
How to tackle the diagnosis of limb-girdle muscular dystrophy $2 \mathrm{~A}$
}

\author{
Marina Fanin ${ }^{* 1,2}$, Anna Chiara Nascimbeni ${ }^{1,2}$, Elisabetta Tasca ${ }^{1,2}$ and Corrado Angelini ${ }^{1,2}$ \\ ${ }^{1}$ Department of Neurosciences, University of Padova, Padova, Italy; ${ }^{2}$ Venetian Institute of Molecular Medicine, Padova, \\ Italy
}

Limb-girdle muscular dystrophy (LGMD) 2A (calpainopathy) is the most frequent form of LGMD in many European countries. The increasing demand for a molecular diagnosis makes the identification of strategies to improve gene mutation detection crucial. We conducted both a quantitative analysis of calpain-3 protein in 519 muscles from patients with unclassified LGMD, unclassified myopathy and hyperCKemia, and a functional assay of calpain-3 autolytic activity in 108 cases with LGMD and normal protein quantity. Subsequently, screening of CAPN3 gene mutations was performed using allele-specific tests and simplified SSCP analysis. We diagnosed a total of 94 LGMD2A patients, carrying 66 different mutations (six are newly identified). The probability of diagnosing calpainopathy was very high in patients showing either a quantitative $(\mathbf{8 0} \%)$ or a functional calpain-3 protein defect $(88 \%)$. Our data show a high predictive value for reduced-absent calpain-3 or lost autolytic activity. These biochemical assays are powerful tools for otherwise laborious genetic screening of cases with a high probability of being primary calpainopathy. Our multistep diagnostic approach is rational and highly effective. This strategy has improved the detection rate of the disease and our extension of screening to presymptomatic phenotypes (hyperCKemia) has allowed us to obtain early diagnoses, which has important consequences for patient care and genetic counseling.

European Journal of Human Genetics (2009) 17, 598-603; doi:10.1038/ejhg.2008.193; published online 15 October 2008

Keywords: LGMD2A; calpain-3; limb-girdle muscular dystrophy; protein testing; molecular diagnosis

\section{Introduction}

Autosomal recessive limb-girdle muscular dystrophies (LGMD type 2) are a heterogeneous group of disorders, which are characterized by progressive involvement and wasting of proximal limb-girdle muscles, and include at least 14 different genetic entities (LGMD2A-LGMD2N).

Although LGMD2I is the most common form of all LGMDs in Northern Europe, ${ }^{1}$ LGMD2A (MIM\#253600) is the most prevalent in many European countries, ${ }^{2-10}$ Turkey, ${ }^{11,12}$ Brazil, $^{13}$ Japan, $^{14,15}$ Russia $^{16}$ and Australia, ${ }^{17}$

${ }^{*}$ Correspondence: Dr M Fanin, Department of Neurosciences, University of Padova, Venetian Institute of Molecular Medicine, via Giuseppe Orus 2, Padova, PD 35129, Italy.

Tel: + 39049 7923202; Fax: + 39049 7923250;

E-mail: marina.fanin@unipd.it

Received 25 April 2008; revised 29 August 2008; accepted 4 September 2008; published online 15 October 2008 with variable frequencies that differ depending on ethnic clusters and geographic origins. Estimates based on molecular data indicate that LGMD2A frequency ranges from about $10 \%$ of LGMD cases in the United States ${ }^{18,19}$ to $80 \%$ in the Basque country and Russia. ${ }^{16,20}$

LGMD2A is caused by mutations in the CAPN3 gene (MIM\#114240, mapped to 15q15.1-q21.1) encoding for a muscle-specific proteolytic enzyme called calpain- $3^{21}$ that is involved in the complex process of sarcomere remodeling. ${ }^{22}$

Many clinical and molecular studies, carried out on large series of calpainopathy patients, have highlighted the heterogeneous features of the disease at the clinical, molecular and protein levels. ${ }^{23-26}$ The onset of muscle weakness and the progress of clinical course may vary considerably, as may the spectrum of phenotypes that is becoming increasingly wider, including, hyperCKemia, 
pseudometabolic myopathy and eosinophilic myositis. 5,27,28 $^{2}$ Genotype-phenotype correlation studies have demonstrated that this clinical variability may be only partly attributable to gene mutations ${ }^{24,26,29,30}$ (interfamilial and intrafamilial variability because of the same mutation), which suggests that additional epigenetic/environmental factors might play a role in modulating the phenotype expression.

The molecular diagnosis of LGMD2A is increasingly being demanded because of the high frequency of the disease. This diagnosis is challenging, because such mutational analysis is cumbersome as it has to identify widespread mutations in this relatively large gene. Thus, it is essential that strategies, which can detect those muscle biopsies that are likely to harbor CAPN3 gene mutations, as well as improve and simplify mutation detection in general have to be found.

Our approach to the diagnosis of LGMD2A is based on a preliminary quantitative protein analysis in muscle that is associated with a functional assay to assess calpain-3 autolytic activity when the quantity is normal and on subsequent gene mutation screening.

\section{Materials and methods}

\section{Selection criteria of patients and muscle biopsies}

The muscle biopsy bank at the Neuromuscular Center in the University of Padova, which contains about 8000 specimens, was surveyed to select patients who fulfiled the following criteria:

- normal expression of dystrophin, $\alpha$-sarcoglycan, dysferlin, caveolin-3, merosin, emerin in muscle biopsy using immunoblot or immunohistochemical analysis;

- muscle histopathology consistent with a dystrophic or myopathic process;

- clinical phenotype ranging from LGMD to asymptomatic with only increased serum creatine kinase (CK) level at rest ( $>500 \mathrm{U} / \mathrm{l})$ of unknown etiology.

Of 519 patients who were included in this study, 242 had LGMD, 90 had unclassified myopathy and 187 had asymptomatic hyperCKemia. Fifty-eight LGMD2A patients included in this study have been described previously (Table 1).

At the time of diagnosis, open biopsies of quadriceps femoris or biceps brachii muscle were obtained after written informed consent from patients or their relatives. All procedures were conducted after the approval had been obtained from the University review board.

\section{Semiquantitative immunoblot analysis of calpain-3}

Conventional immunoblot analysis of calpain-3 was conducted as described previously ${ }^{23}$ with minor modification. Briefly, cryostat sections of muscle biopsies were dissolved in loading buffer, boiled and centrifuged. Proteins were resolved by SDS-polyacrylamide gel electrophoresis and blotted to nitrocellulose membrane. Blots were air-dried, blocked with 5\% nonfat milk in Tris-Tween-20 saline buffer and incubated overnight with monoclonal antibodies against calpain-3 (Calp12A2 - epitope in exon 8 Novocastra Laboratory, Newcastle upon Tyne, UK) diluted 1:800. After thorough washing, the immunoreactive bands were visualized using antimouse peroxidase-conjugated antibodies and the chemioluminescent method (ECL, GE Healthcare, UK).

The quantity of calpain-3 protein in the patient's sample was determined by densitometry using Image software v.1.34 (normalizing the full-sized 94-kDa band on blots to the myosin band in the post-transfer Coomassie bluestained gels), and was expressed as a percentage of control.

We arbitrarily considered the reduction in calpain-3 protein quantity of less than $50 \%$ of controls as a significant deficiency (partial protein defect).

\section{Analysis of calpain-3 autolytic activity}

The muscle biopsies from patients who showed normal calpain-3 quantity with preliminary immunoblot, underwent a further in vitro biochemical assay that was developed using a modified version of the conventional immunoblot to test calpain-3 autocatalytic activity. ${ }^{31}$ In brief, muscle cryostat sections from controls and patients were quickly dissolved in saline solution and incubated at room temperature for $5 \mathrm{~min}$, before the reaction was blocked by adding loading buffer containing EDTA chelating $\mathrm{Ca}^{++}$ions. All samples were then processed as described for conventional calpain-3 immunoblot.

Mutation analysis by allele-specific tests - ARMS-PCR (amplification refractory mutation system)

Genomic DNA was extracted from leukocytes or muscle tissue. To quickly test a number of mutant alleles, more frequently occur in Italian and European populations, ${ }^{5,24,26}$ we selected 17 different mutations: c.550delA, p.G222R, p.R448H, p.R572W, p.R748Q, p.R748X, p.D77N, p.R440Q, p.R110X, p.D753N and p.V354G were analyzed using classic ARMS-PCR test, whereas p.R448C, p.R489Q, p.R490W, p.R490Q, p.G496R and p.P82 L were tested using tetra-primer ARMS-PCR.

In the tetra-primer ARMS-PCR method, one pair of outer control primers and two inner primers specific for mutant or wild-type allele are used in the same reaction. By positioning the outer primers at different distances from mutation point, two different small allele-specific products and one large control PCR product are produced. Primer sequences (Supplementary Table 1) were designed using the human CAPN3 sequence as reference (GenBank accession \#AF209502.1) and Primer-Express software for ARMS-PCR or a specific software (http://cedar.genetics.soton.ac.uk/public_html/primer1.html) for tetra-primer ARMS. PCR reactions were performed as described elsewhere. ${ }^{31}$ 
Table 1 Clinical, biochemical and molecular data in 94 LGMD2A patients

\begin{tabular}{|c|c|c|c|c|c|c|}
\hline Patient number & Phenotype & Calpain quantity (\%) & Calpain autolytic activity & Mutant allele 1 & Mutant allele 2 & Reference \\
\hline 1 & LGMD & 0 & - & c.967G > T, p.E323X, ex.7 & c. $1303 \mathrm{G}>\mathrm{A}, \mathrm{p} . \mathrm{E} 435 \mathrm{~K}$, ex. 10 & Fanin et $a P^{26}$ \\
\hline 2 & LGMD & $<5$ & - & c. $328 \mathrm{C}>T, p . R 110 X$, ex.2 & c. $649 \mathrm{G}>\mathrm{A}, \mathrm{E} 217 \mathrm{~K}$, ex.5 & Fanin et $a a^{26}$ \\
\hline $\begin{array}{l}3 \\
4\end{array}$ & $\begin{array}{l}\text { LGMD } \\
\text { LGMD }\end{array}$ & $\begin{array}{l}0 \\
0\end{array}$ & $=$ & $\begin{array}{l}\text { c.5900G>A, p.R197H, ex.4 } \\
\text { c.550delA ex.4 }\end{array}$ & 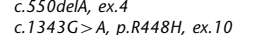 & 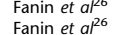 \\
\hline 5 & LGMD & 0 & - & c.533T>C, p.1178T, ex.4 & c. $533 \mathrm{~T}>\mathrm{C}$, p.1178T, ex. 4 & Fanin $e t a l^{26}$ \\
\hline 6 & LGMD & 0 & - & c.550delA, ex.4 & c. $2330 \mathrm{~T}>\mathrm{C}$, p. $1777 \mathrm{~T}$, ex. 22 & Fanin et $a l^{26}$ \\
\hline 7 & hyperCkemia & 0 & - & c. $1992+1 \mathrm{C}>\mathrm{T}$, intr. 17 & c. $1193+6 \mathrm{~T}>\mathrm{A}$, intr.9 & Fanin et $a^{26}$ \\
\hline 8 & LGMD & 0 & - & c. $1333 \mathrm{G}>\mathrm{A}, \mathrm{p} . \mathrm{G} 445 \mathrm{R}, \mathrm{ex} .10$ & & Fanin et $a^{26}$ \\
\hline 9 & LGMD & 0 & - & c.550delA, ex.4 & c.550delA, ex.4 & Fanin et $a a^{26}$ \\
\hline 10 & LGMD & 0 & - & c. $550 \mathrm{delA}$, ex. 4 & c.550delA, ex.4 & Fanin et $a a^{26}$ \\
\hline $\begin{array}{l}11 \\
12\end{array}$ & $\begin{array}{l}\text { LGMD } \\
\text { LGMD }\end{array}$ & $\begin{array}{l}5 \\
0\end{array}$ & - & $c .229 \mathrm{G}>A, p . D 77 N$, ex. 1 & & Fanin et al ${ }^{26}$ \\
\hline $\begin{array}{l}12 \\
13\end{array}$ & $\begin{array}{l}\text { LGMD } \\
\text { LGMD }\end{array}$ & $\begin{array}{l}0 \\
0\end{array}$ & $=$ & $\begin{array}{l}\text { c.1992+1G>T, intr.17 } \\
\text { c.1342C }>T \text {, p.R448C ex.10 }\end{array}$ & $\begin{array}{l}c .1061 T>G, p . V 354 C, \text { ex. } 8 \\
C .1342 C>I\end{array}$ & $\begin{array}{l}\text { Fanin et } a l^{26} \\
\text { Eanin } a^{26}\end{array}$ \\
\hline 14 & LGMD & 0 & - & 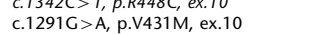 & $c .1343 C>T, p . R 448 C$, ex. 10 & $\begin{array}{l}\text { Fanin ex et } \\
\text { Fanin et al }\end{array}$ \\
\hline 15 & LGMD & 0 & - & c. $1621 \mathrm{C}>\mathrm{T}$, p.R541W, ex.13 & c.598_612del, ex.4 & Fanin et $a l^{26}$ \\
\hline 16 & LGMD & 0 & - & c.1792_1795delAAAA, ex.15 & c. $802-9 \mathrm{G}>\mathrm{A}$, intr. 5 & Fanin et $a l^{26}$ \\
\hline 17 & LGMD & 0 & - & c.139_141delATC, p.147del, ex.1 & c.139_141delATC, p.147del, ex.1 & Present study \\
\hline 18 & LGMD & 0 & - & $\begin{array}{l}c .550 \text { delA, ex.4 } \\
\text {. }\end{array}$ & c. $229 \mathrm{G}>A, p . D 77 \mathrm{~N}$, ex. 1 & Fanin et $a^{26}$ \\
\hline $\begin{array}{l}19 \\
20\end{array}$ & $\begin{array}{l}\text { LGMD } \\
\text { LGMD }\end{array}$ & $\begin{array}{l}0 \\
0\end{array}$ & - & $\begin{array}{l}c .245 \mathrm{C}>T, p . P 82 L, \text { ex. } 1 \\
\text { c5sodelA }\end{array}$ & $\begin{array}{l}\text { C.697G > C, p.G233R, ex.5 } \\
\text { C 550delA ex. }\end{array}$ & $\begin{array}{l}\text { Fanin et al }{ }^{26} \\
\text { Eanin }{ }^{26}\end{array}$ \\
\hline 21 & LGMD & 0 & $\overline{-}$ & $\begin{array}{l}\text {.c5sodela, ex.4 } \\
\text { c.139-141delATC, p.147del, ex.1 }\end{array}$ & $\begin{array}{l}\text { C.S3OdelA exx.4 } \\
\text { c.139_141delATC, p.147del, ex.11 }\end{array}$ & $\begin{array}{l}\text { Fanin et al- } \\
\text { Present study }\end{array}$ \\
\hline 22 & LGMD & 0 & - & c. $848 \mathrm{~T}>$ C, p.M283T, ex.6 & c. $848 \mathrm{~T}>\mathrm{C}$, p.M283T, ex.6 & Fanin et $a l^{26}$ \\
\hline 23 & LGMD & 0 & - & c. $1319 \mathrm{G}>A, p . R 440 \mathrm{Q}$, ex.10 & c. $1343 \mathrm{G}>A, p . R 448 H$, ex.10 & Fanin $e t a l^{26}$ \\
\hline 24 & LGMD & $<5$ & - & $c .229 \mathrm{G}>A, p . D 77 \mathrm{~N}, \mathrm{ex.} .1$ & c.590G > A, p.R197H, ex.4 & Fanin et $a^{26}$ \\
\hline 25 & LGMD & 0 & - & c.550delA, ex.4 & c. $801+1 \mathrm{G}>\mathrm{A}$, intr.5 & Fanin et $a l^{26}$ \\
\hline $\begin{array}{l}26 \\
27\end{array}$ & $\begin{array}{l}\text { LGMD } \\
\text { LGMD }\end{array}$ & $\begin{array}{l}0 \\
0\end{array}$ & $=$ & c.550delA, ex.4 & 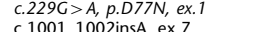 & Fanin et $a^{26}$ \\
\hline $\begin{array}{l}27 \\
28\end{array}$ & $\begin{array}{l}\text { LGMD } \\
\text { LGMD }\end{array}$ & $\begin{array}{l}0 \\
0\end{array}$ & - & 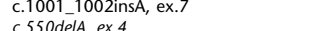 & 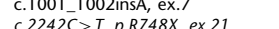 & $\begin{array}{l}\text { Present study } \\
\text { Eanin } e^{2 t} a^{26}\end{array}$ \\
\hline 29 & LGMD & 5 & $\overline{-}$ & c.1401_1403delGGA, p.E467del.ex.11 & $c .2242 C>T, p . R 748 x$, ex. 21 & $\begin{array}{l}\text { ranin et al } \\
\text { Fanin et } a^{26}\end{array}$ \\
\hline 30 & hyperCkemia & $<5$ & - & c. $1385 \bar{T}>\mathrm{G}, \mathrm{L} 462 \mathrm{R}, \mathrm{ex} .11$ & - & Present study \\
\hline 31 & LGMD & 0 & - & $c .2242 C>T, p . R 748 X, e x .21$ & & Fanin et $a l^{26}$ \\
\hline 32 & LGMD & 0 & - & c. $1343 \mathrm{G}>A, p . R 448 \mathrm{H}$, ex.10 & c. $1992+1 \mathrm{G}>\mathrm{T}$, intr. 17 & Fanin et $a l^{26}$ \\
\hline $\begin{array}{l}33 \\
34\end{array}$ & LGMD & $\begin{array}{r}0 \\
-5\end{array}$ & - & c.550delA, ex.4 & c.550delA, ex.4 & Fanin et $a^{26}$ \\
\hline $\begin{array}{l}34 \\
35\end{array}$ & $\begin{array}{l}\text { LGMD } \\
\text { LGMD }\end{array}$ & $\begin{array}{r}<5 \\
0\end{array}$ & - & $\begin{array}{l}\text { c.550dede, ex.4 } \\
\text { c.100dele, ex.1 }\end{array}$ & $\begin{array}{l}c .550 \mathrm{del} A \mathrm{~A}, \text { ex.4 } \\
c .228 \mathrm{~A}>\mathrm{CG} \text {. } Y 763 \mathrm{C} \text { ex.22 }\end{array}$ & $\begin{array}{l}\text { Fanin et } a l^{26} \\
\text { Eanin } a^{26}\end{array}$ \\
\hline 36 & LGMD & $<5$ & - & $\begin{array}{l}\text { c.l.100deli, ex.I } \\
\text { c. } 1345 \mathrm{~A}>\mathrm{C} \text {, p.N449H, ex. } 10\end{array}$ & 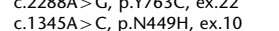 & $\begin{array}{l}\text { Faninin et t } t^{26} \\
\text { Fanin } a l^{2}\end{array}$ \\
\hline 37 & LGMD & 0 & - & c.139_141delATC, p.147del, ex.1 & c.139_141delATC, p.147del, ex.1 & Present study \\
\hline 38 & LGMD & 0 & - & $c .2243 \mathrm{G}>A, p . R 748 Q$, ex.21 & $c .2243 G>A, p . R 748 Q$, ex.21 & Fanin et $a l^{26}$ \\
\hline 39 & LGMD & 0 & - & c.610C > G, p.L204V, ex.4 & c. $1746-20 \mathrm{C}>\mathrm{C}$, intr. 13 & Present study \\
\hline $\begin{array}{l}40 \\
41\end{array}$ & hyperCkemia & 5 & - & c. 550 delA, ex.4 & c. $1746-20 \mathrm{C}>\mathrm{G}$, intr. 13 & $\begin{array}{l}\text { Fanin et a a } a^{16} \\
\text { Eani }\end{array}$ \\
\hline $\begin{array}{l}41 \\
42\end{array}$ & $\begin{array}{l}\text { LGMD } \\
\text { hyperCkemia }\end{array}$ & $\begin{array}{r}0 \\
<5\end{array}$ & $=$ & 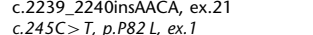 & c.2239_2240insAACA, ex.21 & $\begin{array}{l}\text { Fanin et a } \text { al }^{26} \\
\text { Presenty study }\end{array}$ \\
\hline 43 & LGMD & 0 & - & $\begin{array}{l}\text { c.24sc>l, p.prozL, ex.I } \\
\text { c.550delA, ex.4 }\end{array}$ & c.1030-1G >A, intr.7 & $\begin{array}{l}\text { Presenen study } \\
\text { Present study }\end{array}$ \\
\hline 44 & LGMD & 0 & - & c.550delA, ex.4 & & Present study \\
\hline 45 & LGMD & 0 & - & $c .2243 G>A, p . R 748 Q, e x .21$ & $c .2243 G>A, p . R 748 Q, e x .21$ & Present study \\
\hline 46 & LGMD & 0 & - & c.550delA, ex.4 & c.550delA, ex.4 & Present study \\
\hline 47 & LGMD & 5 & - & c. $1524+1 \mathrm{G}>\mathrm{C}$, intr.11 & c. $755 \mathrm{~T}$ > C, p.M252T, ex.5 & Present study \\
\hline $\begin{array}{l}48 \\
49\end{array}$ & LGMD & 5 & - & 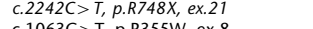 & $\begin{array}{l}c .2257 \mathrm{G}>A, p . D 753 \mathrm{~N}, \text { ex.21 } \\
\text {. }\end{array}$ & $\begin{array}{l}\text { Present study } \\
\text { Presents thuydy }\end{array}$ \\
\hline $\begin{array}{l}49 \\
50\end{array}$ & $\begin{array}{l}\text { hyperckemia } \\
\text { LGMD }\end{array}$ & $\begin{array}{l}0 \\
0\end{array}$ & $=$ & $\begin{array}{l}\text { c.1063C>T, p.R355W, ex.8 } \\
\text { c.2257G>>, p.D753N, ex.21 }\end{array}$ & c.1063C>T, p.R355W, ex.8 & $\begin{array}{l}\text { Present study } \\
\text { Fanin } e t a^{2}\end{array}$ \\
\hline 51 & LGMD & 0 & - & c.550delA, ex.4 & c.550delA, ex.4 & $\begin{array}{l}\text { Fanin et al } \\
\text { Present study }\end{array}$ \\
\hline 52 & LGMD & $10-20$ & - & c. $697 \mathrm{G}>$ C, p. G233R, ex. 5 & c. $1746-20 \mathrm{C}>\mathrm{C}$, intr. 13 & Present study \\
\hline 53 & LGMD & 0 & - & c.550delA, ex.4 & c.550delA, ex.4 & Present study \\
\hline 54 & LGMD & 0 & - & c.59delc, ex.1 & c.59delc, ex.1 & Present study \\
\hline 55 & LGMD & 10 & - & $c .229 \mathrm{G}>A, p . D 77 \mathrm{~N}$, ex. 1 & 1706 20s $>c$ intr 13 & Fanin et $a^{26}$ \\
\hline $\begin{array}{l}56 \\
57\end{array}$ & $\begin{array}{l}\text { hyperCkemia } \\
\text { LGD }\end{array}$ & 20 & - & $\begin{array}{l}c .245 \mathrm{C}>T, p . P 82 L, \text { ex. } 1 \\
\text {. }\end{array}$ & $\begin{array}{l}\text { c. } 1746-20 \mathrm{C}>\mathrm{C} \text {, intr.13 } \\
\text {. }\end{array}$ & $\begin{array}{l}\text { Fanin et a } a^{126} \\
\text { Fanin } a^{26}\end{array}$ \\
\hline 58 & $\begin{array}{l}\text { LGMD } \\
\text { LGMD }\end{array}$ & $\begin{array}{l}10 \\
50\end{array}$ & $=$ & & $\begin{array}{l}c .10611>C, p . V 354 C, \text { ex. } \\
c .1309 \mathrm{C}>\mathrm{T} \text { o } \mathrm{R} 437 \mathrm{C} \text { ex. } 10\end{array}$ & $\begin{array}{l}\text { Fanin et } a^{a^{26}} \\
\text { Fanin et a }\end{array}$ \\
\hline 59 & LGMD & 10 & $\overline{-}$ & c. $479 \mathrm{C}>\mathrm{C}$, p. $\mathrm{A} 160 \mathrm{G}$, ex. 3 & c. $1029+3 \mathrm{~A}>\mathrm{G}$, intr. 7 , 10.10 & $\begin{array}{l}\text { Fanin et } a^{26} \\
\text { Fanin et } a l^{26}\end{array}$ \\
\hline 60 & LGMD & 10 & - & c. $1061 T>G, p . V 354 G$, ex.8 & c. $1746-20 C>C$, intr. 13 & Fanin et $a l^{26}$ \\
\hline 61 & LGMD & 80 & - & c. $1061 \mathrm{~T}>$ C, p.V354A, ex.8 & c. $1621 \mathrm{C}>\mathrm{T}, \mathrm{p} . \mathrm{R} 541 \mathrm{~W}, \mathrm{ex} .13$ & Fanin et al 26 \\
\hline & hyperCkemia & 50 & - & c. $755 \mathrm{~T}>\mathrm{G}$, p.M252R, ex. 5 & - & Fanin et $a^{26}$ \\
\hline 63 & $\begin{array}{l}\text { hyperCkemia } \\
\text { L G }\end{array}$ & 20 & $=$ & $\begin{array}{l}\text { c. } 1193+6 \mathrm{~T}>\mathrm{A} \text {, intr.9 } \\
\end{array}$ & - & Present study \\
\hline $\begin{array}{l}64 \\
65\end{array}$ & $\begin{array}{l}\text { LGMD } \\
\text { hyperCkemia }\end{array}$ & $\begin{array}{l}10 \\
10\end{array}$ & $\overline{-}$ & 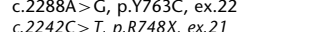 & $\bar{c} .143 G>A, 0.548 N$ ex. 1 & $\begin{array}{l}\text { Fanin et a a }{ }^{16} \\
\text { Fanin et } a^{126}\end{array}$ \\
\hline $\begin{array}{l}63 \\
66\end{array}$ & $\begin{array}{l}\text { nypere kemia } \\
\text { LGMD }\end{array}$ & 10 & $=$ & 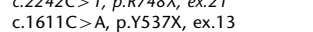 & $\begin{array}{l}c .1430>A, p=547, \text { ex. } \\
.1714 C>T, p . R 572 W, \text { ex. } 13\end{array}$ & $\begin{array}{l}\text { Fanin et } a^{26} \\
\text { Fanin et } a l^{26}\end{array}$ \\
\hline 67 & LGMD & 20 & - & c. $1193+6 \mathrm{~T}>\mathrm{A}$, intr.9 & - & Present study \\
\hline 68 & hyperCkemia & 30 & - & c.539A >C, p.D180A, ex.4 & - & Present study \\
\hline 69 & hyperCkemia & 50 & - & c. $1303 \mathrm{G}>\mathrm{A}$, p.E435K, ex.10 & c. 1193+6T > A, intr.9 & Present study \\
\hline 70 & LGMD & $\begin{array}{l}100 \\
100\end{array}$ & Lost & c. $1469 \mathrm{G}>A, p . R 490 \mathrm{Q}$, ex.11 & $\begin{array}{c}c .1469 \mathrm{G}>A, p . R 490 \mathrm{Q}, \text { ex.11 } \\
\text { ent }\end{array}$ & $\begin{array}{l}\text { Fanin et } a^{26} \\
\text { Presents thudy }\end{array}$ \\
\hline 72 & $\begin{array}{l}\text { LGMD } \\
\text { LGMD }\end{array}$ & $\begin{array}{l}100 \\
100\end{array}$ & $\begin{array}{l}\text { Normal } \\
\text { Lost }\end{array}$ & 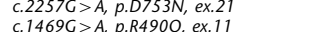 & 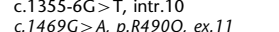 & $\begin{array}{l}\text { Present study } \\
\text { Fanin } e \text { t } a^{26}\end{array}$ \\
\hline 73 & hyperCkemia & 100 & Lost & 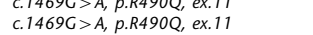 & 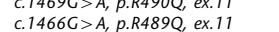 & $\begin{array}{l}\text { Fanin et al } \\
\text { Fanin et } a^{26}\end{array}$ \\
\hline & LGMD & 100 & Lost & c. $1486 \mathrm{C}>A, p . G 496 R$, ex. 11 & c. $984 \mathrm{C}>\mathrm{A}, \mathrm{p} . \mathrm{C} 328 \mathrm{X}$, ex. 7 & Fanin et $a l^{26}$ \\
\hline & LGMD & 100 & Normal & c. $1468 \mathrm{C}>T, p . R 490 \mathrm{~W}$, ex.11 & & Present study \\
\hline 76 & LGMD & 100 & Lost & c. $1468 \mathrm{C}>T, p . R 490 \mathrm{~W}$, ex. 11 & c. $1193+6 \mathrm{~T}>\mathrm{A}$, intr.9 & Fanin et $a^{26}$ \\
\hline & LGMD & 100 & Lost & c. $1469 \mathrm{G}>A, p . R 490 \mathrm{Q}$, ex.11 & $c .1469 \mathrm{G}>A, p . R 490 \mathrm{Q}$, ex.11 & Fanin et $a^{26}$ \\
\hline $\begin{array}{l}78 \\
79\end{array}$ & $\begin{array}{l}\text { LGMD } \\
\text { LGMD }\end{array}$ & $\begin{array}{l}100 \\
100\end{array}$ & $\begin{array}{l}\text { Lost } \\
\text { Lost }\end{array}$ & $\begin{array}{l}c .1469 G>A, p . R 490 Q, \text { ex.11 } \\
c .146 G>A\end{array}$ & c. $1469 \mathrm{G}>A, p . R 490 \mathrm{Q}$, ex.11 & 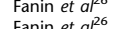 \\
\hline 80 & $\begin{array}{l}\text { LGMD } \\
\text { LGMD }\end{array}$ & $\begin{array}{l}100 \\
100\end{array}$ & $\begin{array}{l}\text { Lost } \\
\text { Normal }\end{array}$ & 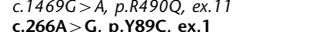 & C.530dela, ex.4 & $\begin{array}{l}\text { Fanin et al } \\
\text { Present study }\end{array}$ \\
\hline 81 & LGMD & 100 & Normal & 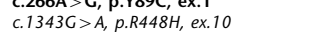 & & Fanin et $a a^{26}$ \\
\hline 82 & LGMD & 100 & Lost & c. $1984 \mathrm{G}>\mathrm{T}$, p.A662S, ex.17 & c.235G > C, p. .79 Q, ex. 1 & Present study \\
\hline 83 & LGMD & 100 & Lost & c.550delA, ex.4 & c. $1468 C>T, p . R 490 W$, ex. 11 & Fanin et $a^{26}$ \\
\hline 84 & LGMD & 100 & Normal & & c.259_260inst, ex.1 & Fanin et $a l^{26}$ \\
\hline 85 & LGMD & 100 & Lost & c. $.575 \mathrm{C}>\mathrm{T}$, p.T1921, ex.4 & c. $1611 \mathrm{C}>\mathrm{A}, \mathrm{p} . \mathrm{Y5} 37 \mathrm{X}$, ex.13 & Present study \\
\hline 86 & LGMD & 100 & Lost & c. $1486 \mathrm{C}>A, p . G 496 R$, ex.11 & & Present study \\
\hline 87 & LGMD & 100 & Lost & c. 479 C > G, p.A160G, ex. 3 & $c .1714 C>T, p . R 572 W$, ex.13 & Present study \\
\hline 88 & LGMD & 100 & Normal & $c .2257 \mathrm{G}>A, p . D 753 \mathrm{~N}$, ex.21 & & Present study \\
\hline 89 & LGMD & 100 & Lost & $c .328 \mathrm{C}>T, p . R 110 X$, ex. 2 & $A, p . G 222 R$, ex.5 & Present study \\
\hline 90 & LGMD & 100 & Lost & c.1469G>A, p.R490Q, ex.11 & c.1469G>A, p.R490Q, ex.11 & Present study \\
\hline 91 & LGMD & 100 & Normal & $c .245 \mathrm{C}>T, p . P 82 L$, ex. .1 & c.533T>C, p. $.1178 \mathrm{~T}$, ex. 4 & Present study \\
\hline & LGMD & 100 & Normal & c. $1468 \mathrm{C}>T, p . R 490 \mathrm{~W}$, ex.11 & $c .2242 C>T, p . R 748 X$, ex.21 & Present study \\
\hline $\begin{array}{l}93 \\
94\end{array}$ & $\begin{array}{l}\text { LGMD } \\
\text { hyperCkemia }\end{array}$ & $\begin{array}{l}100 \\
100-2 x-1\end{array}$ & ND & 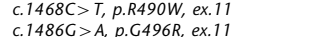 & c.550delA, ex.4 & $\begin{array}{l}\text { Present study } \\
\text { Present study }\end{array}$ \\
\hline & & & & & & \\
\hline
\end{tabular}

ND, not determined. Bold letters indicate newly identified mutations. Italics indicate mutant alleles identified by allele-specific tests. 
Mutation screening by SSCP (single strand conformational polymorphism)

The screening of CAPN3 gene mutations was also performed using SSCP technique. The entire coding sequence (except exons 12 and 24, which are rarely involved in mutations) and the promoter region were amplified by PCR; the products were mixed with denaturing loading buffer, denatured by heating and immediately placed on ice. Gel electrophoresis was conducted using multiple sets of $0.75-\mathrm{mm}$ thick minigels (cells for multiple gels); appropriate acrylamide concentrations (both with and without $5 \%$ glycerol) and electrophoresis conditions were set for each amplicon (Supplementary Table 2). The gels were then silver stained using standard methods.

\section{DNA sequencing and analysis}

The PCR products containing nucleotide changes (distinguishable by aberrant migration bands after SSCP analysis) were purified by enzyme reaction (ExoSap-1, GE Healthcare), and directly sequenced using the Big-Dye di-deoxyterminator cycle sequencing kit and ABI-PRISM 3700 automated sequencer (Applied Biosystems). Sequence analysis was carried out using Chromas and ClustalW softwares with the human CAPN3 gene sequence as reference. The prediction of the potential pathogenetic effect of newly identified mutations was determined by in silico analysis using specific softwares (Splicing Sequence Finder www.umd.be/HSF/; SpliceSiteFinder; BDGP at www.fruitfly.org/seq_tools/splice.html and SIFT at http:// blocks.fhcrc.org/sift/SIFT.html).

\section{Results \\ Calpain-3 quantitative and functional protein screening}

We found a complete or partial protein defect in 87 of the 519 patients selected for the preliminary quantitative calpain-3 protein screening by western blotting. Following this screening, we conducted a functional test of calpain-3 autolytic activity in 108 patients who showed normal calpain-3 quantity and LGMD phenotype. Seventeen cases showed the loss of autolytic function.

\section{CAPN3 gene mutation analysis}

Screening of mutations was performed by allele-specific tests and SSCP analysis in a total of 282 patients: 87 with quantitative protein defect, 17 with loss of autolytic activity and 178 with normal protein quantity (selected on the basis of LGMD phenotype or very high CK levels and showing either normal or unknown autolytic activity).

We identified 66 different mutations (Table 1), 42 (64\%) of missense type, two (3\%) in-frame deletion and 22 (33\%) of null type (nonsense, frame-shifting deletion/insertion, splicing mutations). Most mutations found have already been reported, and some of them recurred in our population. However, six mutations (c.1385T $>$ G, p.L462R exon 11; c.266A > G, p.Y89C exon 1; c.59delC exon 1; c.1030-
$1 \mathrm{G}>\mathrm{A}$ intron $7 ;$ c.235G $>$ C, p.E79Q exon 1 and c. $1193+6 \mathrm{~T}>\mathrm{A}$ intron 9) were identified for the first time (not found in 100 control chromosomes), and their pathogenetic effect has been analyzed.

The c.59delC mutation (patient 54) causes a frameshifting with consequent deleterious effect. The deleterious effect of the c.1030-1G >A mutation (found in patient 43 in heterozygote state with a null mutation and associated with absent protein), is expected. Indeed, in silico analysis predicted that it potentially interferes with the correct splicing by weakening the acceptor site, likely resulting in an out-of-frame skip of exon 8. The c. $1193+6 \mathrm{~T}>\mathrm{A}$ mutation was associated with absent protein, when in heterozygote state with a splice site mutation (patient7), with severe protein defect, when in heterozygote state with a missense (patient 69), or an unknown mutation (patients 63 and 67), or with normal protein quantity, when in heterozygote state with a missense mutation, that causes the loss of autolytic activity (patient 76). These data suggested its deleterious effect. In silico analysis predicted that this mutation potentially interferes with the correct splicing, making more probable a different donor site localized 31 bases downstream the canonical one.

Of the three newly identified missense mutation, the p.E79Q was associated in one sporadic patient (patient 82) with another missense allele and loss of autolytic activity; the p.L462R was found in one patient (patient 30) with $<5 \%$ residual protein quantity, in heterozygote state with an unknown mutation; the p.Y89C was found in one LGMD patient (patient 80 ) with normal protein quantity, in heterozygote state with an unknown mutation. The amino-acid change lysine (nonpolar) to arginine (polar, charged) resulting from p.L462R mutation is expected to severely compromise the structure/function of the protein, whereas the change glutamic acid (polar, charged) to glutamine (polar, uncharged) resulting from p.E79Q mutation and the tyrosine (polar, uncharged) to cysteine (uncharged, nonpolar) resulting from p.Y89C are expected to be more tolerated, as predicted by in silico analysis.

In a total of 94 LGMD2A patients (Table 1), we were able to identify $90.4 \%$ of the mutant alleles, $52 \%$ of which were detected using allele-specific tests and the remainder using SSCP analysis (Table 1).

Of the 17 mutations selected for allele-specific tests, the p.R490Q, p.R490W, p.R489Q, p.G496R and p.G222R were found to be associated with normal protein expression.

Both mutant alleles were identified in $81 \%$ of cases and only one mutant allele was found in $19 \%$ of cases. Although the pathological nature of the second allele remains to be demonstrated, we infer that these latter patients are indeed affected with LGMD2A because they have either a clinical LGMD phenotype, and/or reduced absent protein or loss of calpain-3 autolytic function. The identification of the second mutant allele was likely missed because of the incomplete sensitivity of screening methods 
and the possibility that some mutations may be localized in noncoding regions (eg, deep intron mutations, deletions or duplications of one or more exons).

\section{Molecular characterization of patients}

We diagnosed 94 LGMD2A patients (including 36 newly reported cases, Table 1): $73 \%$ had quantitative protein defect, $16 \%$ had a functional protein defect and $11 \%$ had normal protein quantity.

CAPN3 gene mutations were found in $80 \%$ of the cases showing a complete or partial calpain-3 protein defect and in $88 \%$ of the cases, showing the loss of calpain-3 autolytic function. In two patients (12\%), a false-negative result was obtained with the autolytic activity assay. The combined biochemical-molecular diagnostic approach that we used resulted in a very high probability of obtaining LGMD2A diagnosis in patients who already show either a quantitative or a functional calpain-3 protein defect.

Of the 178 cases with LGMD, myopathy or hyperCKemia of unknown origin who showed normal protein quantity and normal or unknown autolytic activity, molecular screening permitted the identification of a further 10 patients, which indicates that normal finding of calpain-3 quantity is associated with a $5.6 \%$ residual probability of primary calpainopathy.

\section{Discussion}

Since its first description in 1995, a number of studies have investigated LGMD2A at the clinical, biochemical and molecular level to assess the natural history of the disease, to report the atypical clinical features and to correlate the phenotype with the protein expression and the genotype.

In the early years of calpainopathy research, the identification of CAPN3 gene mutations was also performed to investigate the pathogenetic effects of gene mutations, which justified using advanced technical methods and expensive procedures. Recently, laboratories dedicated to neuromuscular diagnosis have been faced with increasing demand for molecular characterization of LGMD2A, largely because of the high frequency of this disease in most populations. Laboratories worldwide molecularly diagnose LGMD2A using a variety of diagnostic approaches, often depending on different clinical options (to perform a muscle biopsy or not), equipment availability (automated sequencer, DHPLC or electrophoresis apparatus), methods adopted for mutation analysis (sequencing the entire coding gene, screening of mutations by DHPLC or SSCP) and both human and economic resources.

Thus, the best way of making further advances in the molecular diagnosis of LGMD2A has become an important topic. Clearly, the fundamental factor that determines the choice of the diagnostic testing strategy in LGMD2A is whether a muscle biopsy is available or not.
Sequence analysis of the entire CAPN3 gene from genomic DNA seems to be the best diagnostic step in unclassified LGMD patients for whom muscle biopsy is unavailable. As LGMD2A is the most common form of LGMD worldwide and in most European countries, this strategy offers 30-40\% probability of identifying calpainopathy patients.

The availability of a muscle biopsy in unclassified LGMD patients is considered the most important tool in the complex process of diagnosis, because it permits the analysis of a number of the muscle proteins responsible for LGMD. Indeed, in many laboratories, the identification of calpain-3 protein defect in muscle is used to select patients for the subsequent gene mutation analysis. ${ }^{23,25,26}$

By identifying 94 LGMD2A patients, we demonstrated that a molecular diagnosis can be obtained in at least $80 \%$ of patients showing calpain-3 protein deficiency, which suggests that the search for mutations is highly efficient in such cases. The eventual extension of mutation screening to patients with unclassified LGMD and normal calpain-3 protein or unknown protein data would provide a lower rate of molecular diagnosis. The molecular analysis of such patients is actually adopted on a research basis.

However, 25\% of the LGMD2A patients in this study showed normal calpain-3 quantity because of mutations that are expected to result in an inactive enzyme and might affect either the autolytic or the proteolytic activity. ${ }^{31,32}$ Furthermore, we feel that because of its high sensitivity (12\% of false-negative results) and easy procedure, the functional test for calpain-3 autolytic activity is a valuable tool in the diagnostic algorithm of LGMD2A when protein quantity is normal.

The choice of the approach to be used in the search of gene mutations may be population dependent. Indeed, single point mutations recur in specific LGMD2A populations and some exonic regions are more susceptible to harbor disease-causing mutations..$^{5,7-9,12,16,23,24,26}$ In our patient population, the use of allele-specific test for recurrent mutations, proved to be an efficacious strategy that has provided a high mutation detection rate and speeded up the genetic analysis.

The possibility that molecular diagnosis could be obtained at RNA level deserves consideration; however, some mutations cannot be detected at RNA level (including those associated with the nonsense-mediated mRNA decay mechanism), and cDNA analysis cannot substitute genomic analysis because identified mutations must be confirmed at genomic level.

The results of our systematic investigation of a large series of patients showed that although LGMD2A diagnosis is based on genetic testing, biochemical assays make it possible to restrict laborious genetic analysis to a limited number of patients who have a high probability of being affected with primary calpainopathy. Using our combined biochemical-molecular strategy, we improved the detection rate of LGMD2A, expanded the screening to patients 
with asymptomatic hyperCKemia and were often able to obtain an early diagnosis which is very important for genetic counseling.

\section{Acknowledgements}

This study was supported by Telethon-Italy (GTB07001 to CA), Association Française contre le Myopathies (2007.0889/12925 to MF), EuroBioBank network (QLRT2001027769 to C.A.), and Italian Ministry for University and Research (COFIN 2006/062912 to CA).

\section{References}

1 Sveen ML, Schwartz M, Vissing J: High prevalence and phenotype-genotype correlations of limb girdle muscular dystrophy type 2I in Denmark. Ann Neurol 2006; 59: 808-815.

2 Bushby KM, Beckmann JS: The 105th ENMC sponsored workshop: pathogenesis in the non-sarcoglycan limb girdle muscular dystrophies. Neuromusc Disord 2003; 13: 80-90.

3 Fanin M, Nascimbeni AC, Fulizio L, Angelini C: The frequency of limb girdle muscular dystrophy $2 \mathrm{~A}$ in northeastern Italy. Neuromusc Disord 2005; 15: 218-224.

4 Van der Kooi AJ, Frankhuizen WS, Barth PG et al: Limb-girdle muscular dystrophy in the Netherlands: gene defect identified in half the families. Neurology 2007; 68: 2125-2128.

5 Piluso G, Politano L, Aurino $S$ et al: Extensive scanning of the calpain-3 gene broadens the spectrum of LGMD2A phenotypes. J Med Genet 2005; 42: 686-693.

6 Guglieri M, Magri F, D'Angelo MG et al: Clinical, molecular and protein correlations in a large sample of genetically diagnosed Italian limb girdle muscular dystrophy patients. Hum Mut 2008; 29: $258-266$

7 Hanisch F, Muller CR, Grimm D et al: Frequency of calpain-3 c.550delA mutation in limb girdle muscular dystrophy type 2 and isolated hyperCKemia in German patients. Clin Neuropathol 2007; 26: $157-163$.

8 Canki-Klain N, Milic A, Kovac B et al: Prevalence of the 550delA mutation in calpainopathy (LGMD2A) in Croatia. Am J Med Genet 2003; 125: $152-156$.

9 Chrobakova T, Hermanova M, Kroupova I et al: Mutations in Czech LGMD2A patients revealed by analysis of calpain-3 mRNA and their phenotypic outcome. Neuromusc Disord 2004; 14: 659-665.

10 Groen EJ, Charlton R, Barresi R et al: Analysis of the UK diagnostic strategy for limb girdle muscular dystrophy 2A. Brain 2007; 130: 3237-3249.

11 Dincer P, Leturcq F, Richard I et al: A biochemical, genetic, and clinical survey of autosomal recessive limb girdle muscular dystrophies in Turkey. Ann Neurol 1997; 42: 222-229.

12 Balci B, Aurino S, Haliloglu G et al: Calpain-3 mutations in Turkey. Eur J Pediatr 2006; 165: 293-298.

13 Passos-Bueno MR, Vainzof M, Moreira ES, Zatz M: Seven autosomal recessive limb-girdle muscular dystrophies in the Brazilian population: from LGMD2A to LGMD2G. Am J Med Genet 1999; 82: 392-398.

14 Kawai H, Akaike M, Kunishige $M$ et al: Clinical, pathological, and genetic features of limb-girdle muscular dystrophy type $2 \mathrm{~A}$ with new calpain 3 gene mutations in seven patients from three Japanese families. Muscle Nerve 1998; 21: 1493-1501.

15 Minami N, Nishino I, Kobayashi O et al: Mutations of calpain-3 gene in patients with sporadic limb-girdle muscular dystrophy in Japan. J Neurol Sci 1999; 171: 31-37.

16 Pogoda TV, Krakhmaleva IN, Lipatova NA et al: High incidence of 550delA mutation of CAPN3 in LGMD2 patients from Russia. Hum Mut 2000; 15: 295.

17 Lo HP, Cooper ST, Evesson FJ et al: Limb-girdle muscular dystrophy: diagnostic evaluation, frequency and clues to pathogenesis. Neuromusc Disord 2008; 18: 34-44.

18 Moore SA, Shilling CJ, Westra S et al: Limb-girdle muscular dystrophy in the United States. J Neuropathol Exp Neurol 2006; 65: 995-1003.

19 Chou FL, Angelini C, Daentl D et al: Calpain III mutation analysis of a heterogeneous limb-girdle muscular dystrophy population. Neurology 1999; 52: 1015-1020.

20 Urtasun M, Saenz A, Roudaut C et al: Limb girdle muscular dystrophy in Guipuzcoa (Basque Country, Spain). Brain 1998; 121: $1735-1747$.

21 Richard I, Broux O, Allamand Vet al: Mutations in the proteolytic enzyme calpain 3 cause limb-girdle muscular dystrophy type $2 \mathrm{~A}$. Cell 1995; 81: 27-40.

22 Kramerova I, Kudryashova E, Venkatraman G, Spencer MJ: Calpain 3 participates in sarcomere remodelling by acting upstream of the ubiquitin-proteasome proteolytic pathway. Hum Mol Genet 2005; 14: 2125-2134.

23 Anderson LVB, Davison K, Moss JA et al: Characterization of monoclonal antibodies to calpain-3 and protein expression in muscle from patients with limb girdle muscular dystrophy type 2A. Am J Pathol 1998; 153: 1169-1179.

24 Richard I, Roudaut C, Saenz A et al: Calpainopathy. A survey of mutations and polymorphisms. Am J Hum Genet 1999; 64: $1524-1540$

25 Pollitt C, Anderson LVB, Pogue R et al: The phenotype of calpainopathy: diagnosis based on a multidisciplinary approach. Neuromusc Disord 2001; 11: 287-296.

26 Fanin M, Fulizio L, Nascimbeni AC et al: Molecular diagnosis in LGMD2A: mutation analysis or protein testing? Hum Mut 2004; 24: $52-62$.

27 Penisson-Besnier I, Richard I, Dubas F et al: Pseudometabolic expression and phenotypic variability of calpain deficiency in two siblings. Muscle Nerve 1998; 21: 1078-1080.

28 Krahn M, Lopez de Munain A, Streichenberger N et al: CAPN3 mutations in patients with idiopathic eosinophilic myositis. Ann Neurol 2006; 59: 905-911.

29 Saenz A, Leturcq F, Cobo AM et al: LGMD2A: genotypephenotype correlations based on a large mutational survey on the calpain-3 gene. Brain 2005; 128: 732-742.

30 De Paula F, Vainzof M, Passos-Bueno MR et al: Clinical variability in calpainopathy: what makes the difference? Eur J Hum Genet 2002; 10: 825-832.

31 Fanin M, Nascimbeni AC, Angelini C: Screening of calpain-3 autolytic activity in LGMD muscle: a functional map of CAPN3 gene mutations. J Med Genet 2007; 44: 38-43.

32 Milic A, Daniele N, Lochmuller $\mathrm{H}$ et al: A third of LGMD2A biopsies have normal calpain-3 proteolytic activity as determined by an in-vitro assay. Neuromusc Disord 2007; 17: $148-156$.

Supplementary Information accompanies the paper on European Journal of Human Genetics website (http://www.nature.com/ejhg) 